\title{
THE DAVID DUNLAP OBSERVATORY (DDO) PHOTOMETRIC SYSTEM FOR CLASSIFICATION OF LATE-TYPE STARS
}

\author{
R. D. MCCLURE \\ Dept. of Astronomy, Yale University Observatory, U.S.A.
}

\begin{abstract}
An intermediate bandpass filter system is described that is useful for classification of $G$ and $\mathrm{K}$ stars in terms of effective temperature (spectral type), surface gravity (luminosity class), heavy element abundance, and interstellar reddening. The system measures the strength of the cyanogen band in the region shortward of $\lambda 4216$, the break in the continuum at the $G$ band, and a colour index in the $\lambda 4500-\lambda 4900$ region of the spectrum .The surface gravity parameter is independent of the cyanogen index, and the cyanogen band strength, after a surface gravity correction, is used as a measure of heavy element abundance.

Application of the system to the determination of the atmospheric parameters of open cluster giants, as well as of field stars, is discussed. The system has been used to isolate stellar populations among field stars without any prior knowledge of their distances. From observations on this system, the only open cluster found so far to have giants with stronger cyanogen band absorption than the Hyades giants is the intermediate age southern cluster NGC 2477.
\end{abstract}

\section{Introduction}

The atmospheric parameters that can be measured conveniently in late-type stars are effective temperature, surface gravity and chemical composition. If a photometric system is used, the reddening due to interstellar absorption is usually required before the other parameters can be obtained accurately. The ideal photometric filter system should measure these four parameters with as much sensitivity as possible, with maximum possible transmission of light, and with minimum possible number of filter bandpasses. In practice, a compromise must always be made between maximizing the information gain and minimizing the number of bandpasses, and between sensitivity of the indices versus width of the bandpasses. Generally, the narrower the bandpass, the more sensitive is the photometric index to changes in strength of the spectral feature in question. On the other hand, the advantage of this gain in sensitivity can easily be lost due to a decrease in accuracy of the measurement because less light is transmitted by the narrower filter bandpass.

The photometric system described here was developed with these ideas firmly in mind. Only very coarse spectral features are measured so that the filter bandpasses could be kept relatively wide. As a result, even giant stars in external dwarf galaxies are within reach of at least one existing telescope using this photometric system. The sensitivity of the indices are such, however, that the accuracy of the measurements never has to exceed $\pm 0.01 \mathrm{mag}$.

\section{The Filter Bandpasses}

The filter bandpasses were selected by examination of a comprehensive file of spectrophotometer tracings of late-type stars collected over a number of years by $\operatorname{Dr} \mathrm{S}$. van den Bergh with the David Dunlap Observatory $188-\mathrm{cm}$ reflector. These tracings were 
ideal for the purpose because of the coarse resolution $(\sim 20 \AA)$ of the spectrophotometer. This smeared out the fine features of the spectrum, leaving visible only coarse features that can be measured with filters of reasonable bandwidth. By examination of these tracings, therefore, optimum bandwidths and positions could be determined. The bandpasses chosen are listed in Table I.

TABLE I

DDO filter bandpasses

\begin{tabular}{llc} 
Filter name & Peak wavelength & Bandwidth \\
\hline 48 & $4886 \AA^{\mathrm{a}}$ & $186 \AA$ \\
45 & 4517 & 76 \\
42 & 4257 & 73 \\
41 & 4166 & 83
\end{tabular}

aAn error appears in the 48 filter wavelength in Table XVIII of McClure and van den Bergh (1968).

Measurements using the four filters listed in Table I are used to form conventional colour indices as defined by McClure and van den Bergh (1968). Some characteristics of these indices are as follows:

$C(41-42)$ is a measure of the strength of the cyanogen band absorption in the region shortward of $\lambda 4216$. The strength of this band is known to be very sensitively correlated with luminosity (Lindblad and Stenquist, 1934), and with heavy element abundance (Keenan and Keller, 1953; Griffin and Redman, 1960) of late-type stars.

$C(42-45)$ is a measure of the strength of the break in the continuum at the $\mathrm{G}$ band. This has long been known to be a sensitive measure of effective temperature. For example see the work of Gyldenkerne (1958).

$C(45-48)$ is a colour index devoid of strong spectral features, and has been found emperically to be sensitive to surface gravity in G and K-type stars.

The extinction coefficients for the above three colour indices are very small, making the system very adaptable for observations in poor weather conditions or at poor observing sites. The mean coefficients for the Kitt Peak National Observatory are $0.02,0.05$, and 0.05 for $C(41-42), C(42-45)$, and $C(45-48)$ respectively. The small effect of atmospheric extinction is due, of course, to the fact that the filter bandpasses are separated by only a few hundred Angstroms. The bandpasses are also narrow enough that there are no colour terms in the extinction coefficients for these three indices.

The above indices are useful for the measurement of the atmospheric parameters of Population I stars. In addition, two filter bandpasses in the ultraviolet spectral region were chosen that are a necessary addition when studying extreme Population II stars, and these will be discussed in a subsequent paper by Dr Osborn. From observations with the two ultraviolet filters two additional colour indices may be formed:

$C(38-42)$ is a measure of the discontinuity near $\lambda 4000$ due to metallic line blanketing in the ultraviolet spectral region. 
$C(35-38)$ is a measure of the Balmer discontinuity.

The original set of observations on the system were done by McClure and van den Bergh (1968) using the 188-cm reflector of the David Dunlap Observatory. Altogether, 210 bright stars of a wide range of spectral type, luminosity class and metal abundance, distributed throughout the northern sky were observed. A new set of standards is now being set up in the equatorial region using the facilities of the Kitt Peak National Observatory, to make the system more convenient to the southern hemisphere observer.

\section{The Determination of Reddening}

Young B stars are the most reliable reddening indicators. Often, however, such stars are not available near the object of interest. This is particularly the case in old stellar populations such are found at high galactic latitudes and in old open clusters. In these cases, it is much more convenient if reddening can be determined using red giant stars which are usually the brightest stars in the population.

The DDO system, combined with the $B-V$ colour index of the $U B V$ system has been used successfully to determine reddening of red giant stars in a number of fields. This is possible because the $\mathrm{G}$ band break index $C(42-45)$ has about equal sensitivity to effective temperature as the wide band $B-V$ index whereas the separation in wavelength of the filter bandpasses, and therefore the effect of interstellar reddening, is only about one quarter that of $B-V$. McClure and Racine (1969) have developed a method for obtaining reddening, from DDO photometry of red giants, resulting in the following equation:

$$
E(B-V)=2.175(B-V)-2.380 C(45-48)-1.420 C(42-45)+1.841 .
$$

The values of reddening computed from this equation are applicable for $\mathrm{G}$ and $\mathrm{K}$ giants in the colour range $0.80<(B-V)_{0}<1^{\mathrm{m}} .55$, luminosity class (LC) in the range II $<\mathrm{LC}<\mathrm{IV}$, and for stars of a moderate range of metal abundance. The random error of an individual reddening determination has been found to be a few hundredths of a magnitude, and therefore, with only a few stars an accurate mean value of reddening is obtained. Values of reddening determined from DDO photometry to date are given in Table II.

TABLE II

Reddening values from DDO photometry

\begin{tabular}{lll}
\hline Field & $E(B-V)$ & Reference \\
M3 & 0.00 & McClure and Racine (1969) \\
M13 & 0.00 & McClure and Racine (1969) \\
M31 & 0.11 & McClure and Racine (1969) \\
M33 & 0.03 & McClure and Racine (1969) \\
N. Gal. Pole & 0.00 & McClure and Crawford (1971) \\
M67 & 0.06 & Janes (1971) \\
NGC 2360 & 0.09 & McClure (1971a) \\
NGC 3680 & 0.09 & McClure (1971a) \\
NGC 2477 & 0.31 & Hartwick, Hesser and McClure (1971)
\end{tabular}


Reddening corrections can be made to the DDO colour indices by applying the following colour excess ratios:

$$
\frac{E C(41-42)}{E(B-V)}=0.066 ; \quad \frac{E C(42-45)}{E(B-V)}=0.234 ; \quad \frac{E C(45-48)}{E(B-V)}=0.354 .
$$

\section{Spectral Classification from DDO Indices}

The $C(45-48)$ index, when plotted vs the $\mathrm{G}$ band break index $C(42-45)$, has been found empirically to show good surface gravity dependence for late-type stars. This is illustrated in Figure 1 where luminosity class Ib, III and V field stars are plotted. The spectral classification of a late-type star observed on the DDO system can be determined from its position in Figure 2 where the indices have been calibrated in terms of spectral type and luminosity class. Since the blanketing lines in this figure are almost parallel to the intrinsic sequences, the classification is insensitive to differences in metal abundance.

Calibration of the position in Figure 1 in terms of effective temperature and surface gravity is more difficult due to the uncertainties in model atmosphere and binary star

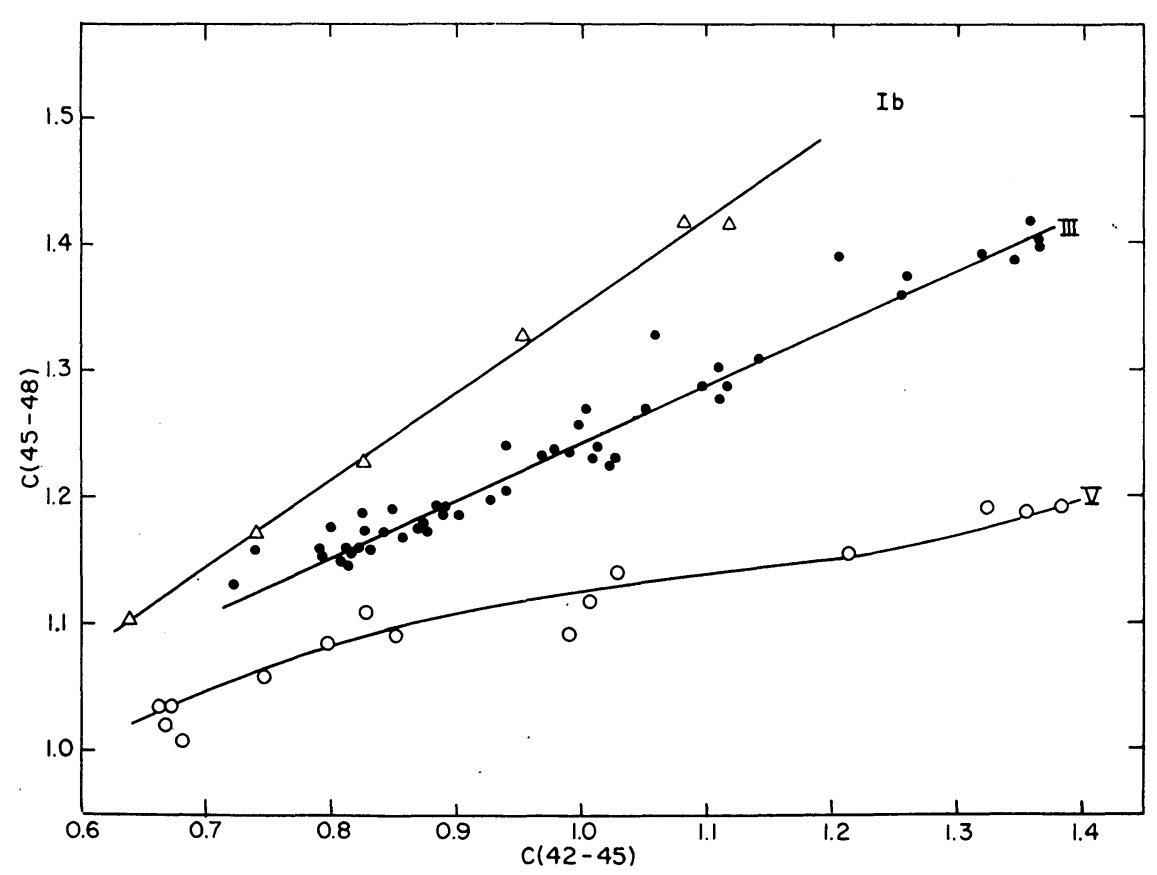

Fig. 1. A plot of the surface gravity dependent colour index $C(45-48)$ vs the temperature dependent colour index $C(42-45)$ for a random sample of late-type luminosity class III stars (dots), class V stars (open circles), and class Ib stars (triangles). The class III stars are all brighter than $V=4 \cdot 0$, and therefore, should have no reddening. Reddening corrections have been applied to the supergiants, however. 


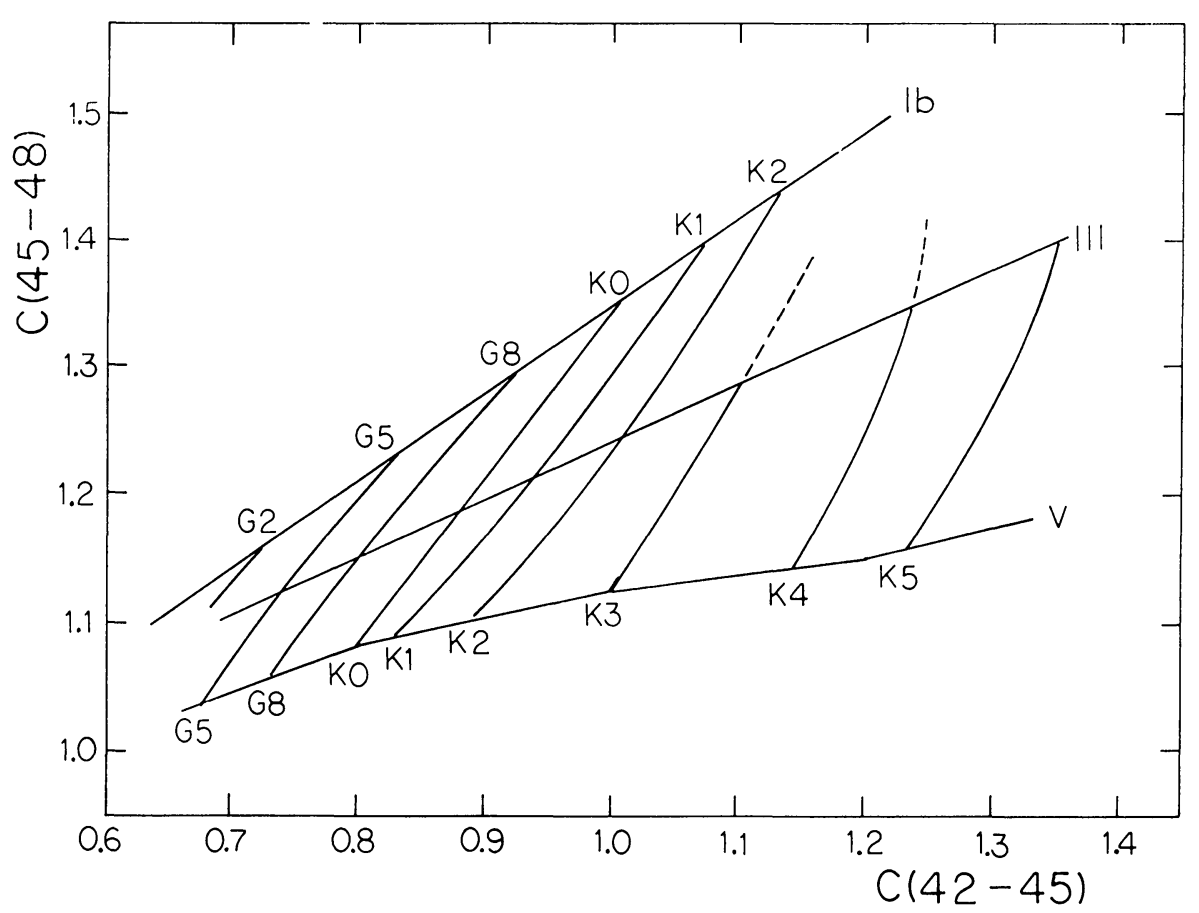

Fig. 2. The calibration of the indices of Figure 1 in terms of spectral type and luminosity class. The blanketing lines are almost parallel to the intrinsic sequences in this diagram.
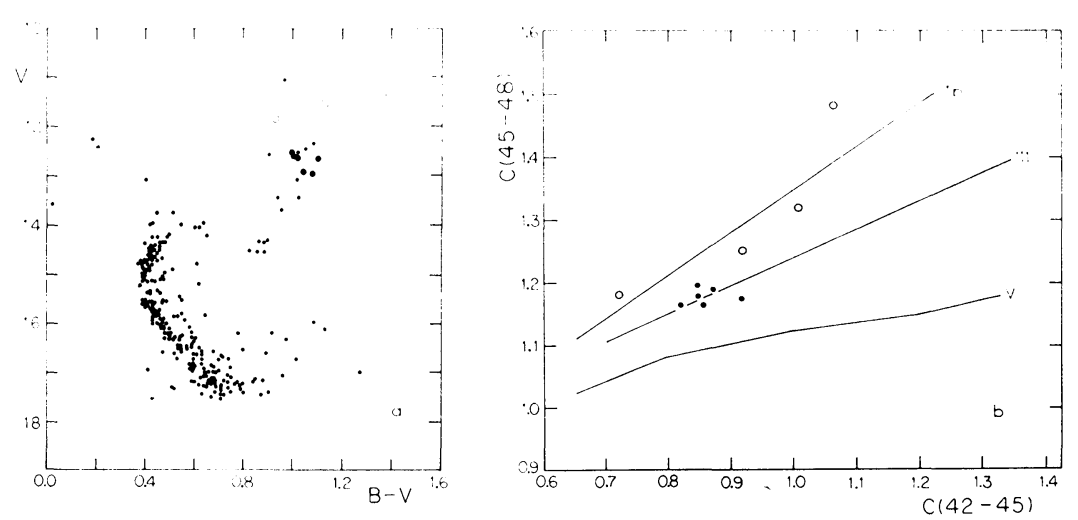

Fig. 3. (a) The colour-magnitude diagram of the old open cluster NGC 2420 from preliminary photographic photometry of Yale 40-in. plates by McClure (1971b). The large dots are stars on the giant branch that were observed on the DDO system, and the open circles are stars that appear above the giant branch that also have DDO photometry. (b) The $C(45-48)$ vs $C(42-45)$ diagram for the same NGC 2420 stars. The four stars that lie above the giant branch in (a) show very low surface gravities in (b). 
results for late-type stars. In addition, blanketing corrections must be applied for this calibration. This will be discussed in a subsequent paper by Osborn. The value of the DDO indices in studying surface gravities of giant stars is shown in Figure 3 where giants in the old open cluster NGC 2420 are shown. Figure 3 a shows the colour-magnitude diagram of the cluster prepared from preliminary photographic photometry with the Yale 40-in. telescope. The stars plotted as large dots and open circles also have DDO photometry, and are shown in the DDO surface gravity diagram in Figure $3 \mathrm{~b}$. The DDO indices indicate that the very luminous giants (open circles) in the colour-magnitude diagram have particularly low surface gravities. This is expected if they have masses equal to or lower than those of the fainter giant branch stars.

\section{The Cyanogen Band Strength}

The $\mathrm{CN}$ band shortward of $\lambda 4216$ is very sensitive to both surface gravity and chemical composition in late-type stars, and therefore, to be useful as a classification parameter, the effects of both these variables must be considered together. For example, the $\mathrm{CN}$ band cannot be used as a surface gravity index without a prior knowledge of the metal abundance of the star and vice versa. In the DDO system, a separate surface

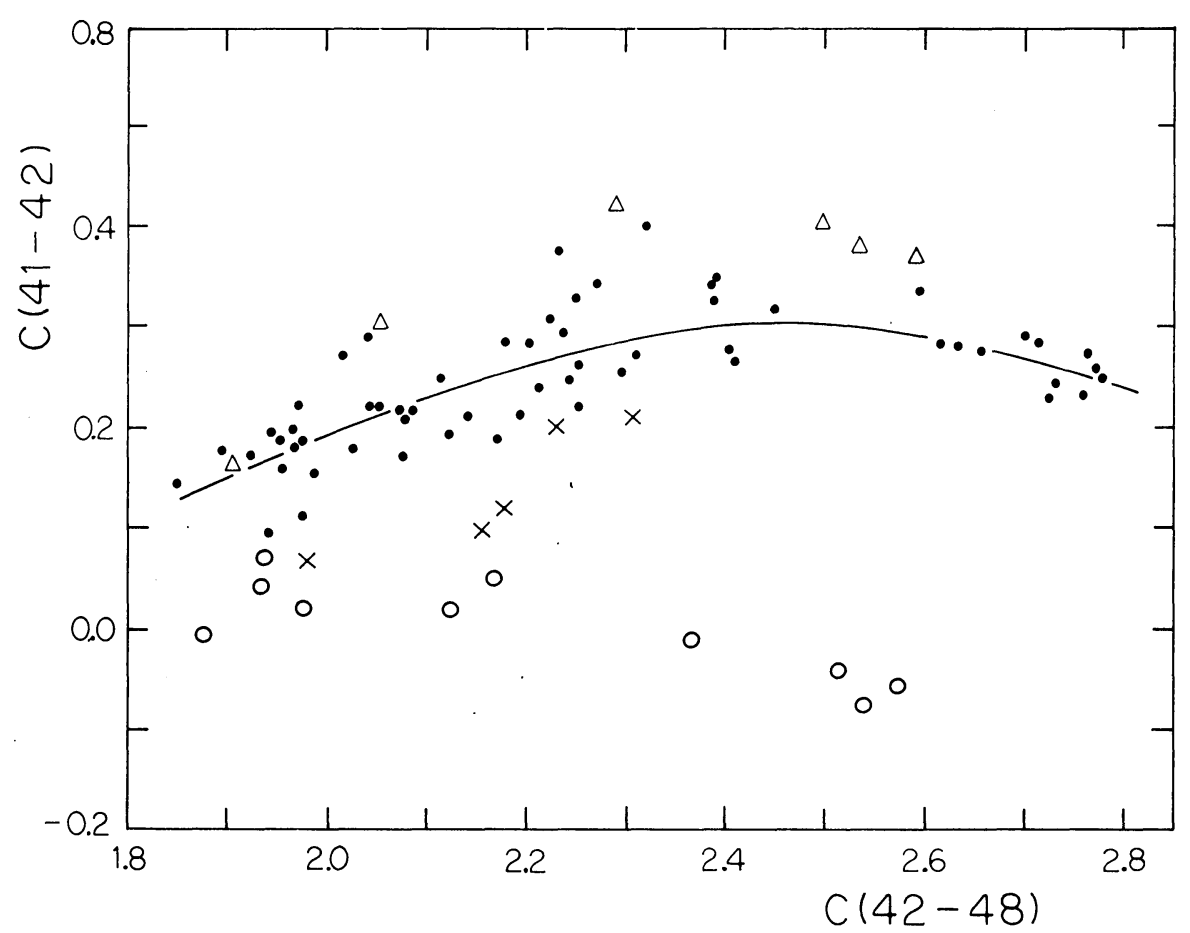

Fig. 4. The $\mathrm{CN}$ index $C(41-42)$ versus the colour index $C(42-48)$ for the random sample of bright luminosity class III stars (dots), class V stars (open circles), and class Ib stars (triangles). Also shown are a few luminosity class III stars that have space velocities greater than $100 \mathrm{~km} \mathrm{~s}^{-1}$ (crosses). 
gravity index already exists, as discussed in the last section. Therefore, advantage is taken of the excellent sensitivity of the $\mathrm{CN}$ band to abundance of the elements in the star, and the band is used as a metallicity parameter after correction for the effects of surface gravity.

Figure 4 shows the $\mathrm{CN}$ index $C(41-42)$ vs the temperature index $C(42-48)$ for field stars near the Sun. Also included are a few high velocity giants that have DDO photometry. Two features to note in this diagram are (1) there is a strong dependence on metal abundance shown by the position of the high velocity giants, and (2) there is a strong dependence on surface gravity. In fact, the latter dependence resembles closely the dependence shown in Figure 1. Because of this resemblance, a simple surface gravity correction can be made to the $\mathrm{CN}$ index of Figure 4 by using the residual from
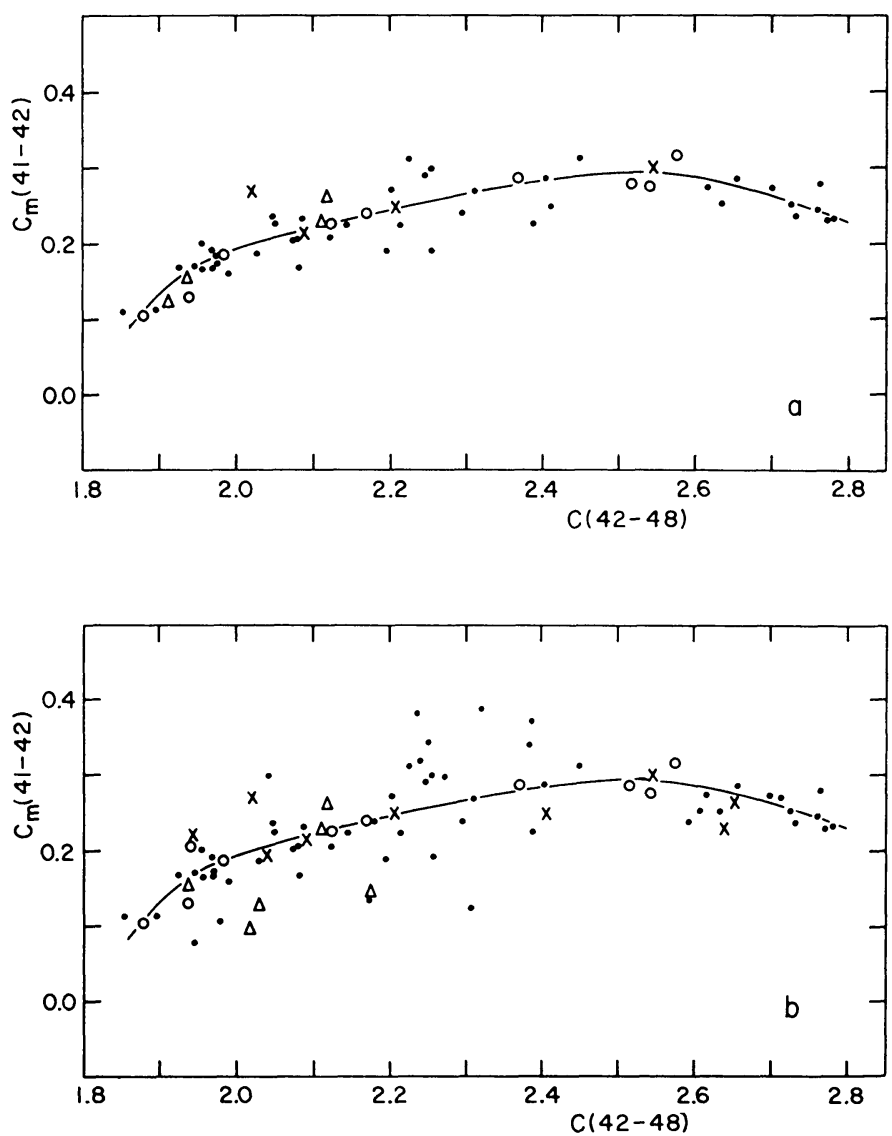

Fig. 5. (a) A plot of the surface gravity free $\mathrm{CN}$ index $C_{m}(41-42)$ vs the colour index $C(42-48)$. Included are bright luminosity class III stars (dots), class II-III and II stars (crosses), class III-IV and IV stars (triangles), and class V stars (open circles). The sample is limited to stars with ultraviolet excesses in the range -0 m.05 $\leqslant \delta(U-B) \leqslant 0$ m.05. (b) The same plot as (a), but with no limitation as to ultraviolet excess. As in Figure 5, there appears to be no separation of luminosity classes, but the increased scatter in this diagram indicates good sensitivity of the index to metallicity. 
the mean class III line in Figure 1. The $\mathrm{CN}$ index is thereby normalized to represent the CN strength of a luminosity class III star of the same metallicity. McClure (1970) has formed a $\mathrm{CN}$ index in this way, that is insensitive to variations of surface gravity. It can be expressed by the equation

$$
C_{m}(41-42)=C(41-42)-1.66[C(45-48)-0.45 C(42-45)-0.792]
$$

and is plotted vs $C(42-48)$ in Figure 5. Figure 5a is limited to stars with $|\delta(U-B)|$ $\leqslant 0$. 05 , whereas Figure $5 \mathrm{~b}$ includes all bright stars observed regardless of ultraviolet excess. There appears to be no dependence on surface gravity but the increased scatter in Figure $5 \mathrm{~b}$ is an indication that the index is indeed sensitive to metallicity. This is further illustrated in Figure 6, where a sample of strong $\mathrm{CN}$ stars that have been selected spectroscopically by Schmitt (1971) as exhibiting strong CN bands, plus a number of high velocity stars are plotted.

The residual above or below the mean line drawn in Figure 5 is a measure of metallicity and can be correlated with spectroscopically determined $[\mathrm{Fe} / \mathrm{H}]$ values. This correlation is shown in Figure 7. The scatter is well within the errors expected from the accuracy of the parameters plotted. As illustrated by this figure, the $\mathrm{CN}$ strength is very useful for studying metallicities of population I stars. It is not so useful for

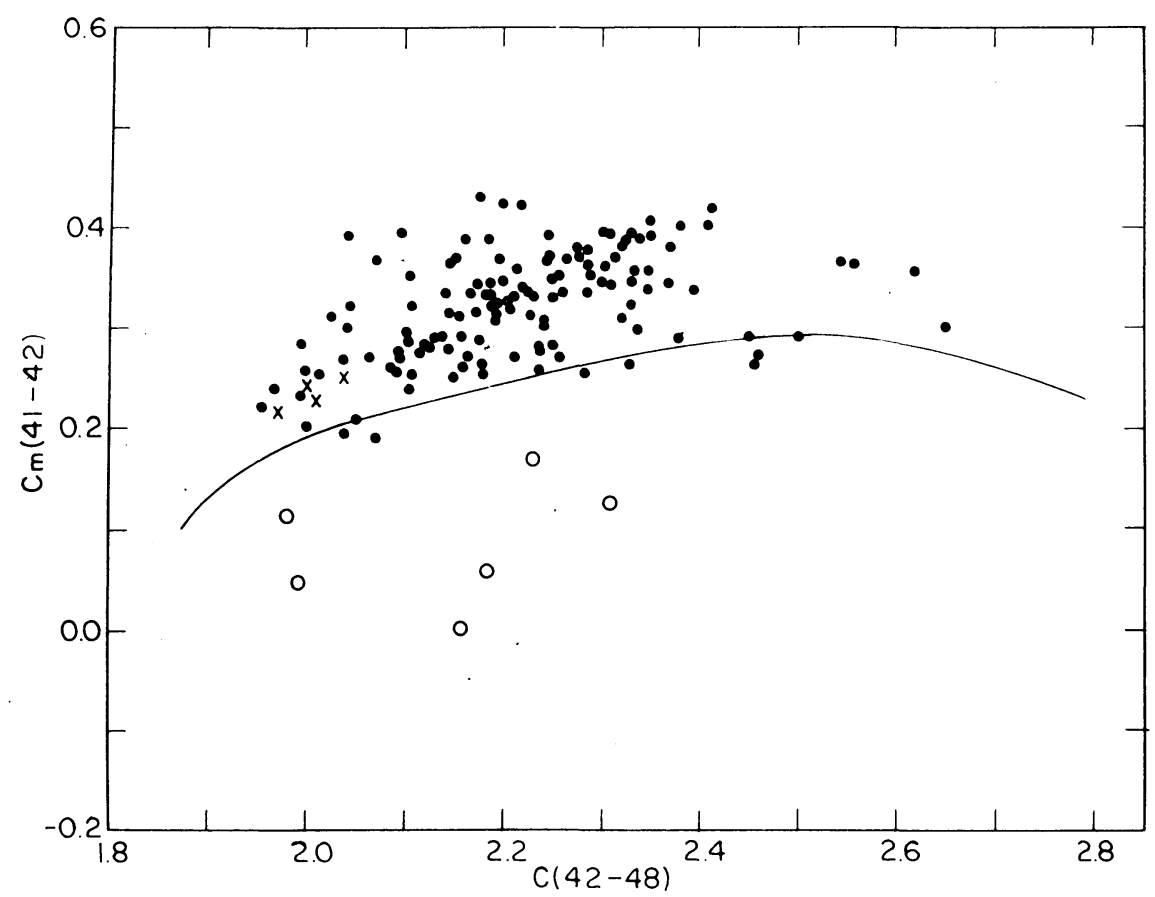

Fig. 6. A plot of $C_{m}(41-42)$ vs $C(42-48)$ for a sample of spectroscopically selected strong CN giants (dots), a sample of high-velocity giants (open circles), and the four Hyades giants (crosses). 


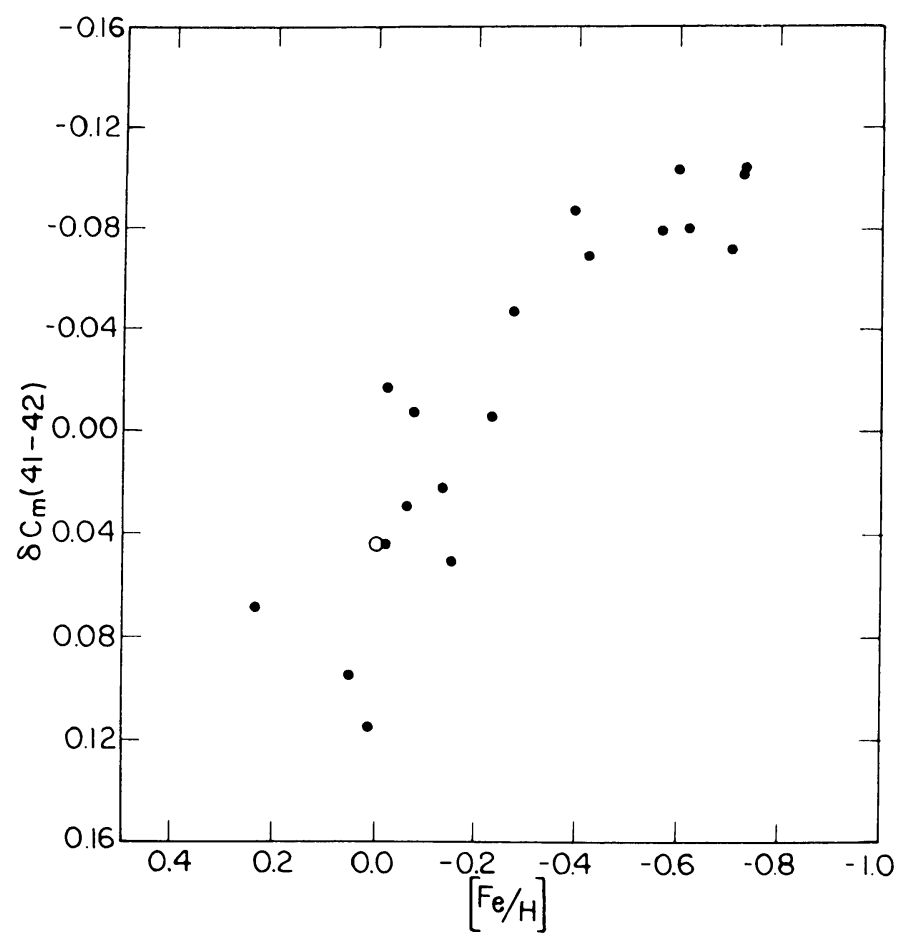

Fig. 7. A correlation between the $\mathrm{CN}$ residual $\delta C_{m}$ and logarithmic iron-to-hydrogen ratios for giant stars from the list published by Wallerstein and Helfer $(1966)$. The $[\mathrm{Fe} / \mathrm{H}]$ values are with respect to the Hyades giants, and the open circle in this figure represents a mean of the four Hyades giants.

halo populations, on the other hand, because the $\mathrm{CN}$ band is very weak in such stars.

\section{Application to Population Studies of Field Stars}

When dealing with field giants one quite often has no prior knowledge of the distance of the stars, and the Population I mass luminosity law does not necessarily hold. The DDO system is very useful for studying field stars, therefore, because it gives a direct indication of surface gravity, temperature, and metallicity without any prior knowledge of the above two parameters.

DDO photometry has been applied recently by McClure and Crawford (1971) to the study of $\mathrm{G}$ and $\mathrm{K}$ giant stars at the north galactic pole. The DDO surface gravity diagram for all late-type stars down to 13th apparent magnitude in a region within a few degrees of the pole is shown in Figure 8. One would expect that in a high latitude field the stellar population would be predominately old. Indeed, Figure 8 resembles the HR diagram of an old cluster. There are pronounced giant and dwarf sequences with subgiants between. In other words, a type of H-R diagram has been constructed for field stars without any knowledge as to their distances. One feature to note here is that there are very few stars of luminosity class brighter than class III. Osborn (1971) 


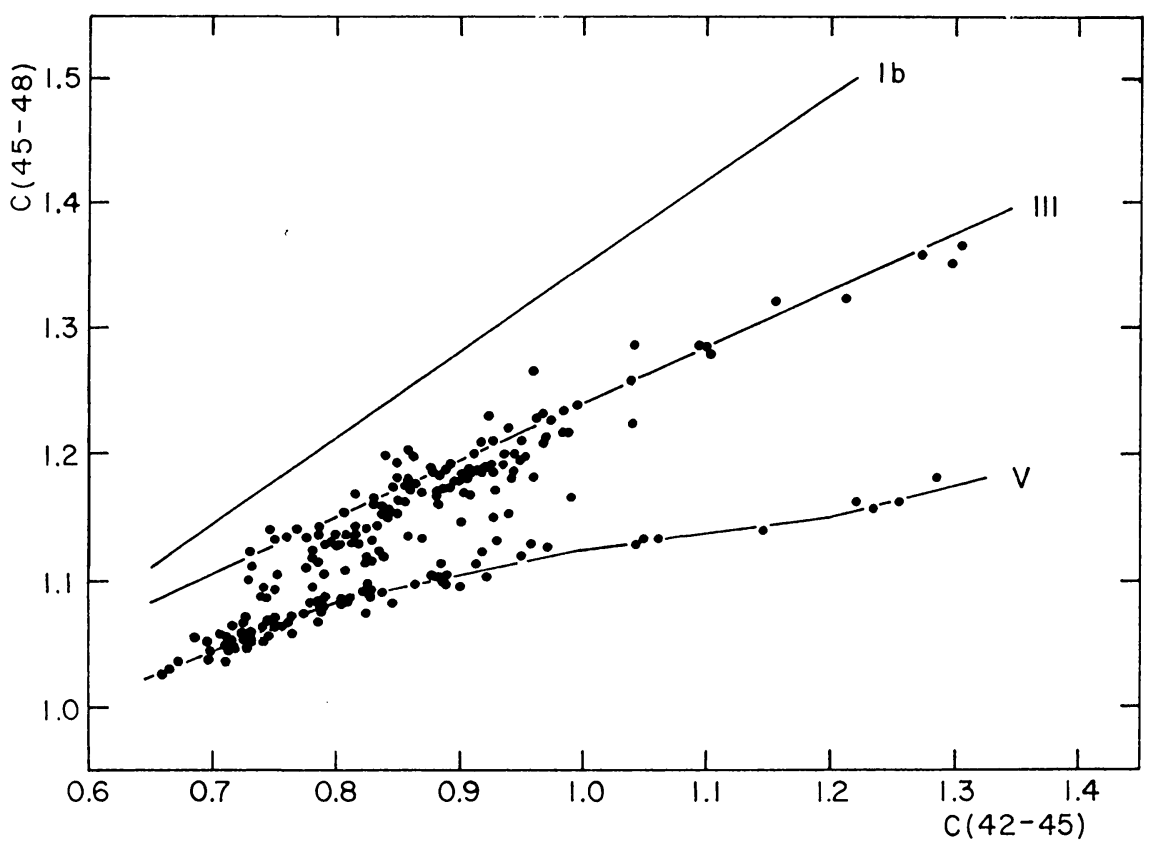

Fig. 8. A plot of the colour index $C(45-48)$ vs the colour index $C(42-45)$ for late-type field star. near the north galactic pole. A main sequence and giant branch extend to late spectral types. Numerous subgiants are found earlier than $\mathrm{K} 1[C(42-45)<0$ m95]. The figure resembles the colourmagnitude diagram of an old open cluster, and this ' $H R$ ' diagram was obtained without any prior knowledge of the distances to these stars.

has found that halo-type stars, as expected due to their low masses, exhibit very low surface gravities in this diagram. Therefore, the sample of stars shown here appears to be predominantly disc population resembling an old open cluster, even though the stars extend to well over a kiloparsec above the galactic plane. The absence of halo stars appears to be due to the sharp fall-off of star numbers along the giant branches of globular clusters near the blue limit of the present study. There is still some question, as pointed out by Helfer and Sturch (1971), as to the calibration at the blue end of Figure 8 in terms of position in a $U B V$ colour-magnitude diagram. In particular, it appears that stars on the horizontal part of the subgiant branch in an old cluster such as M67 (subgiants with $C(42-45)<0$ m 8 ) may fall close to the dwarf sequence.

Figure 9 shows the cyanogen residual $\delta C_{m}$ plotted for the NGP stars vs $Z$ distance determined from the spectral classification of Figure 2 . The metallicity appears to weaken with distance above the galactic plane as expected, but there is large scatter, and one or two anomalous strong $\mathrm{CN}$ stars were found at large distances above the plane.

A sample of bright field stars near the Sun has also been studied by Janes and McClure (1971) to see whether strong CN stars have different kinematics than normal solar neighbourhood giants. The kinematics for the two groups are shown in Figure 10. 


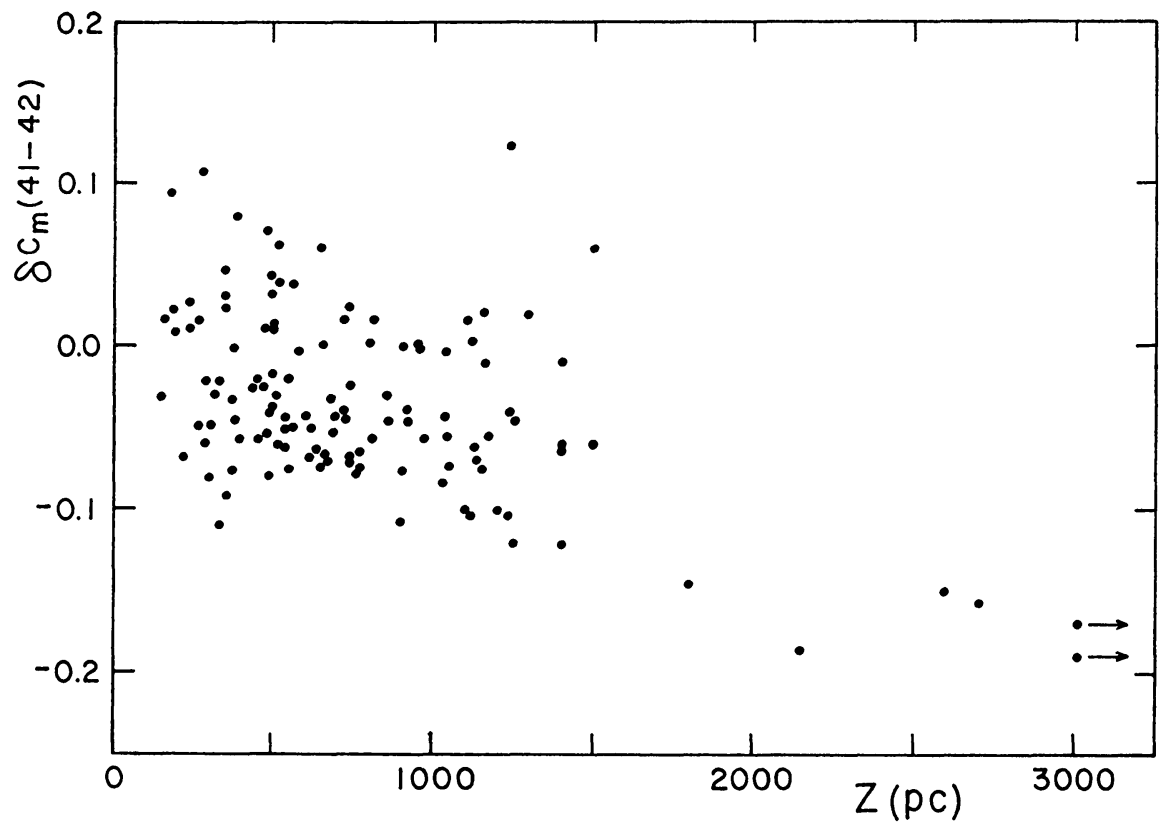

Fig. 9. The $\mathrm{CN}$ residual $\delta C_{m}$ for giant stars in the north galactic pole sample versus distance above the galactic plane.

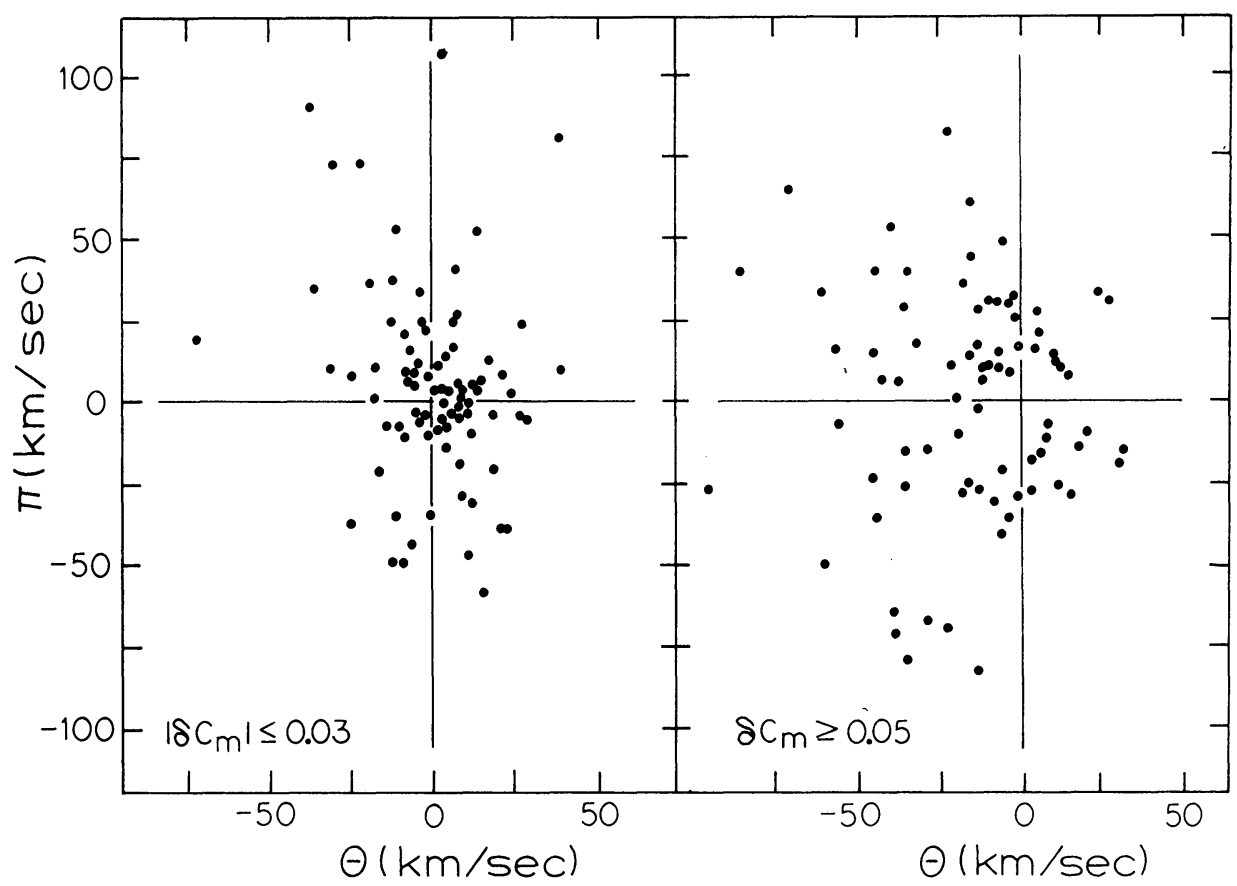

Fig. 10. The distribution of space velocities of field $\mathrm{G}$ and $\mathrm{K}$ giants in the $(\pi, \theta)$ plane. 'Normal stars, are on the left (a), and strong $\mathrm{CN}$ stars are on the right (b). 
The normal giants in Figure $10 \mathrm{a}\left(\left|\delta C_{m}\right| \leqslant 0^{m} .03\right)$ show a concentration of velocities, as expected, near the local standard of rest. The strong CN stars in Figure 10b, on the other hand $\left(\delta C_{m} \geqslant 0.05\right)$ show no such concentration. In this diagram, there appears to be a clumpy distribution forming somewhat of a ring around the local standard of rest, and an underlying widely scattered distribution including some relatively high velocity stars. The clumpy distribution resembles a relatively young population, presumably giants that have evolved from main sequence A-type stars such as Hyades or younger-type giants. In fact, NGC 2477, a southern cluster similar in age to the Hyades has been found from DDO photometry by Hartwick, Hesser and McClure (1971) to have $\mathrm{CN}$ strong stars. The underlying scattered distribution of stars has a mean $\theta$ velocity that is negative, and resembles an old stellar population. There may be a second explanation for these stars, however. They could be the tail end of a population of giants with less energetic orbits, in the mean, than the Sun's. From observations of McClure (1969), Spinrad, Gunn, Taylor, McClure, and Young (1971) of galaxy nuclei, and from Searle's (1971) observations of H II regions in galaxies, it is probable that the metallicity of the Galaxy increases towards the center. Therefore, it is conceivable that $\mathrm{K}$ giant stars whose orbits bring them closer to the center of the Galaxy than the Sun's orbit would have stronger $\mathrm{CN}$ bands than normal.

\section{Application to the Study of Open Clusters}

Red giant stars are the brightest and therefore the most easily observed stars in old open clusters. The giants in several old to intermediate age clusters have now been observed on the DDO system. An example of the DDO surface gravity diagram for the old metal poor cluster NGC 2420 has been shown in Section 4. Observations of a more normal old cluster M67 are shown in Figure 11. Here the coolest giants and
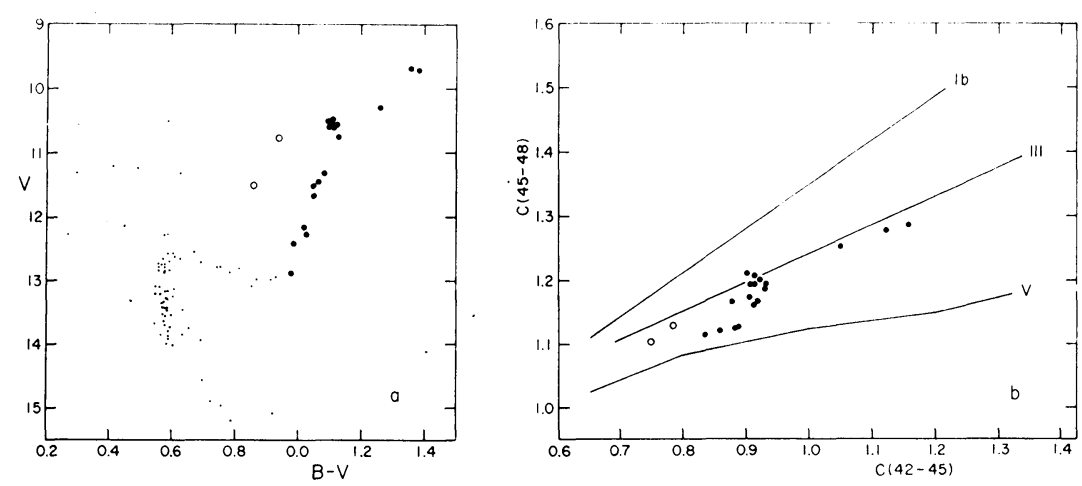

Fig. 11. (a) The colour-magnitude diagram for M67. The large dots are giant stars that have been observed on the DDO system. The open circles are stars lying above the subgiant branch that also have DDO photometry. (b) Preliminary values from Janes (1971) for the $C$ (45-48) versus $C(42-45)$ diagram of giants in M67. The lower luminosity giants show high surface gravities in this diagram, and the two stars that lie above the subgiant branch in the colour-magnitude diagram have lower surface gravities than subgiants. 
clump stars resemble field luminosity class III stars whereas fainter stars on the sloping part of the giant branch in the colour magnitude diagram fall well below the class III relation.

Table III shows the mean CN residuals $\delta C_{m}$ for the giant stars in old and intermediate age clusters observed on the DDO system to date. The clusters are arranged in order of age.

TABLE III

$\mathrm{CN}$ strengths for open clusters

\begin{tabular}{lrrrrl} 
Cluster & \multicolumn{1}{c}{$l^{\mathrm{II}}$} & \multicolumn{1}{c}{$b^{\mathrm{II}}$} & $r(\mathrm{pc})$ & \multicolumn{1}{c}{$\delta C_{m}$} & Reference \\
\hline & & & & & \\
NGC 5822 & $322^{\circ}$ & $+4^{\circ}$ & 700 & $0 \mathrm{~m} 01$ & McClure (1971b) \\
NGC 2477 & 254 & -6 & 1300 & 0.08 & Hartwick et al. \\
Hyades & 180 & -22 & 40 & 0.04 & McClure and van den Bergh (1968) \\
NGC 2360 & 230 & -1 & 1100 & -0.01 & McClure (1971a) \\
NGC 2420 & 198 & +20 & 2600 & -0.07 & McClure (1971b) \\
NGC 3680 & 287 & +17 & 800 & 0.02 & McClure (1971a) \\
NGC 6819 & 74 & +8 & 2000 & 0.01 & McClure (1971b) \\
M67 & 216 & +32 & 800 & 0.04 & Janes (1971)
\end{tabular}

Of particular interest are NGC 2477 and NGC 2420. NGC 2477 is a cluster of about Hyades age (Hartwick et al., 1971) and is situated very close to the galactic plane. It is the only cluster found so far to have giant stars with stronger $\mathrm{CN}$ residuals than the Hyades giants. NGC 2420 is a cluster that appears from its colour-magnitude diagram to be slightly younger than M67, but lies more than $1 \mathrm{kpc}$ above the galactic plane. As well as the giants having weak $\mathrm{CN}$ bands, the main sequence stars in Arp's photoelectric sequence for this cluster have a mean ultraviolet excess of $\delta(U-B)=0$ m. 09 . All other clusters studied to date, including M67, appear to have giants with DDO $\mathrm{CN}$ indices in the range between the Hyades value and the mean of the solar neighbourhood giants.

\section{Acknowledgements}

I would like to acknowledge the support and initial direction of Dr S. van den Bergh who was instrumental in choosing the filter bandpasses for the DDO system and overseeing the development of the standard system of stars. Since the initial observing time allotted at the David Dunlap Observatory for setting up the standard stars, most of the observations have been made at the Kitt Peak National Observatory and Cerro Tololo Inter-American Observatory where the resident astronomers have been very generous in allotment of telescope time and overall support of the project. Observations made with the Yale 40-in. telescope by Mr K. A. Janes have also been very helpful in the preparation of this paper.

\section{References}

Griffin, R. F. and Redman, R. O.: 1960, Monthly Notices Roy. Astron. Soc. 120, 287. 
Gyldenkerne, K.: 1958, Ann. Astrophys. 21, 77.

Hartwick, F. D. A., Hesser, J. E., and McClure, R. D.: 1971, in press.

Helfer, H. L. and Sturch, C.: 1971, private communication.

Janes, K. A.: 1971, in preparation.

Janes, K. A. and McClure, R. D.: 1971, Astrophys. J. 165, 561

Keenan, P. C. and Keller, G.: 1953, Astrophys. J. 117, 241.

Lindblad, B. and Stenquist, E. 1934, Stockholm Obs. Ann. 11, No. 12.

McClure, R. D.: 1969, Astron. J. 74, 50.

McClure, R. D.: 1970, Astron. J. 75, 41.

McClure, R. D.: 1971a, in press.

McClure, R. D.: 1971b, unpublished.

McClure, R. D. and Bergh, S. van den: 1968, Astron. J. 73, 313.

McClure, R. D. and Crawford, D. L.: 1971, Astron.J. 76, 31.

McClure, R. D. and Racine, R.: 1969, Astron.J. 74, 1000.

Osborn, W. H.: 1971, unpublished Ph. D. Thesis, Yale University.

Schmitt, J. L.: 1971, Astrophys. J. 163, 75.

Searle, L. T.: 1971, Carnegie Inst. Washington, Yearb. 69, 75.

Spinrad, H., Gunn, J. E., Taylor, B. J., McClure, R. D., and Young, J. W.: 1971, Astrophys.J. 164, 11. Wallerstein, G. and Helfer, H. L.: 1966, Astron. J. 71, 350. 